\title{
Genetically modified Schwann cells producing glial cell line-derived neurotrophic factor inhibit neuronal apoptosis in rat spinal cord injury
}

\author{
GUOMIN LIU ${ }^{1}$, XUKAI WANG ${ }^{1,2}$, GUOXI SHAO ${ }^{1}$ and QINYI LIU ${ }^{1}$ \\ ${ }^{1}$ Department of Orthopedic Surgery, The Second Hospital of Jilin University, Changchun, Jilin 130041; \\ ${ }^{2}$ Department of Orthopedic Surgery, The Affiliated Hospital to Changchun University of Chinese Medicine, \\ Changchun, Jilin 130021, P.R. China
}

Received June 24, 2013; Accepted January 31, 2014

DOI: $10.3892 / \mathrm{mmr} .2014 .1963$

\begin{abstract}
Schwann cells (SCs) are the major cells constituting the peripheral nerve structure and function, and also secret a variety of neurotrophic factors. Schwann cell (SC) transplantation has recently emerged as a promising therapeutic strategy for spinal cord injury (SCI). In the present study, the ability of genetically modified SCs producing high levels of glial cell line-derived neurotrophic factor (GDNF) to promote spinal cord repair was assessed. The GDNF gene was transduced into SCs. The engineered SCs were characterized by their ability to express and secrete biologically active GDNF, which was shown to inhibit apoptosis of primary rat neurons induced by radiation, and upregulate the expression of B-cell lymphoma 2 (Bcl-2) and downregulate the expression of Bcl-2 associated $\mathrm{X}$ protein (Bax) in vitro. Following $\mathrm{SC}$ implantation into the spinal cord of adult rats with SCI induced by weight-drop impact, the survival of rats with transplanted SCs, histology of the spinal cord and expression levels of Bcl-2 and Bax were examined. Transplantation of unmodified and genetically modified SCs producing GDNF attenuated SCI by inhibiting apoptosis via the Bcl-2/Bax pathways. The genetically modified SCs demonstrated markedly improved recovery of SCI as compared with unmodified SCs. The present study combined the outgrowth-promoting property of SCs with the neuroprotective effects of overexpressed GDNF and identified this as a potential novel therapeutic strategy for SCI.
\end{abstract}

\section{Introduction}

Spinal cord injury (SCI) is a worldwide medical problem with an increasing number of incidences. SCI results in necrosis

Correspondence to: Dr Qinyi Liu, Department of Orthopedic Surgery, The Second Hospital of Jilin University, 218 Ziqing Street, Changchun, Jilin 130041, P.R. China

E-mail: liuyedao123@163.com

Key words: Schwann cells, glial cell line-derived neurotrophic factor, spinal cord injury, apoptosis and apoptosis of neurons and glial cells following the primary and secondary sequential injuries, and the latter may be the main cause for the loss of sensory and motoric function (1). Following SCI, syringomyelia and glial scars constitute a mechanical barrier impeding the growth of axons (2), and neuronal apoptosis decreases the self-repair capacity of the spinal cord and shortage of neurotrophic factors (3). Therefore, despite advances in the elucidation of the pathophysiological processes of SCI, no treatment is currently available to restore the injury-induced loss of function, and further investigation is required to determine therapeutic strategies for SCI (4).

Schwann cells (SCs) are the major cells constituting the peripheral nerve structure and function, and also secret a variety of neurotrophic factors, including nerve growth, brain-derived neurotrophic, neurite-promoting and axon-inducing factors $(5,6)$. The transplantation of SCs may potentially improve axon growth and myelination, inhibit glial scar formation and promote the formation of endometrial nerves $(7,8)$. Previous studies reporting the transplantation of $\mathrm{SCs}$ in rodent and primate models have provided significant evidence of their repair potential for SCI $(9,10)$. The development of in vitro systems to harvest and expand human SCs has made them attractive candidates for autologous transplantation in the clinical setting (11). Therefore, SCs are regarded as ideal seed cells for the repair of SCI.

Glial cell line-derived neurotrophic factor (GDNF) was originally identified and cloned from conditioned media derived from the rat glial cell line B49 and demonstrated survival and differentiation effects on cultured midbrain dopaminergic neurons (12). GDNF is a glycosylated homodimer with a molecular mass of $\sim 33-45 \mathrm{kDa}$ and is a distant member of the transforming growth factor- $\beta$ (TGF- $\beta$ ) superfamily (4). This trophic agent has been shown to inhibit cell death of various central and peripheral neuronal cell types under different conditions (13-16). Its neuroprotective activities include enhancing nerve regeneration, promoting neuronal survival and trophic effects on various neuronal populations (17). Administration of GDNF has been shown to promote recovery of SCI (18-21) and peripheral nerve injuries (22) in animal models, and has also been tested for the treatment of Parkinson's disease in clinical trials (23). Moreover, GDNF also negatively regulates 
the effects of substance abuse and presents a potential target for the treatment of drug addiction (24).

Based on these previous studies, the present study sought to investigate whether genetically modified SCs producing GDNF are able to promote spinal cord repair in a rat model of SCI.

\section{Materials and methods}

Establishment of SCs expressing GDNF. The rat SC line RSC96 was purchased from the Shanghai Institute of Biochemistry and Cell Biology (Shanghai, China). The GDNF-expressing plasmid, pEGFP-N1/GDNF, and empty vector, pEGFP-N1, were kindly provided by the Bio-engineering Laboratory of the School of Stomatology, Jilin University (Changchun, China). The cells were routinely cultured at $37^{\circ} \mathrm{C}$ in Dulbecco's modified Eagle medium (DMEM; Gibco-BRL, Life Technologies, Carlsbad, CA, USA) supplemented with $10 \%$ fetal calf serum (FCS; Gibco-BRL), penicillin $(100 \mathrm{U} / \mathrm{ml})$ and streptomycin $\left(100 \mu \mathrm{g} / \mathrm{ml}\right.$ ) in a $\mathrm{CO}_{2}$ incubator (Heraeus; Thermo Fisher Scientific Inc., Hanau, Germany). Cells $\left(2 \times 10^{5} / \mathrm{ml}\right)$ were seeded in 6-well plates (Costar Cell Culture plate; Corning Incorporated, Lowell, MA, USA) and grown to $90 \%$ confluence at which point they were transfected with $5 \mu \mathrm{g}$ plasmid (pEGFP-N1/GDNF or pEGFP-N1) using Lipofectamine ${ }^{\mathrm{TM}}$ (Gibco-BRL). Cells were detached with $0.25 \%$ trypsin (Sigma-Aldrich, St. Louis, MO, USA) following transfection at 10 multiplicity of infection for $72 \mathrm{~h}$ and seeded at a dilution of $1: 4$ in selection medium containing $1.2 \mathrm{mg} / \mathrm{ml}$ neomycin G418 (Gibco-BRL). The SCs transfected with pEGFP-N1/GDNF or pEGFP-N1 were selected after 3 weeks of culture, and named SCs-pEGFP-N1/GDNF or SCs-pEGFP-N1, respectively.

ELISA. The SCs were seeded into 24-well plates $\left(1 \times 10^{6} / \mathrm{ml}\right)$, and cultured in serum-free DMEM at $37^{\circ} \mathrm{C}$. After 24,48 and $72 \mathrm{~h}$, respectively, the supernatants were collected and subjected to the ELISA to measure the levels of GDNF with a GDNF assay kit (Wuhan Boster Biological Technology, Ltd., Wuhan, China) according to the manufacturer's instructions.

Preparation of primary spinal neurons. All the animal experimental protocols were approved by the Animal Ethics Committee of Jilin University College of Pharmacy (Changchun, China). Inbred Wistar rats at 14-15 days of gestation were provided by the Experimental Animal Center of Jilin University (Changchun, China). Primary culture of spinal cord neurons was performed according to the methods previously described (25). Briefly, spinal cords were quickly removed from the fetuses, washed and minced in Hank's Balanced Salt Solution, and then enzymatically digested with $0.25 \%$ trypsin for $20 \mathrm{~min}$ at $37^{\circ} \mathrm{C}$. The cell suspension was triturated and sieved through a $200 \mu \mathrm{m}$ mesh. Following centrifugation (Sigma Laborzentrifugen GmbH, Ostrode, Germany) at $1,000 \mathrm{x}$ for $10 \mathrm{~min}$, the supernatant was discarded and cells were harvested.

In vitro experimental design. The primary spinal cord neurons were suspended in DMEM at $1 \times 10^{6} / \mathrm{ml}$ and seeded into a 24 -well plate. When the cells reached $\sim 80 \%$ confluency, they were exposed to radiation at $100 \mathrm{cGy} / \mathrm{min}$ with a distance of $100 \mathrm{~cm}$ for $24 \mathrm{~h}$. The total dose of radiation was $2 \mathrm{~Gy}$. The cells were then co-cultured with $\mathrm{SCs}$ at a ratio of 3:1. A transwell membrane $(0.45 \mu \mathrm{m}$ pore size; BD Biosciences, San Jose, CA, USA) was used to separate neurons and SCs. Intact neurons and irradiated neurons served as controls. The cells were cultured for $24 \mathrm{~h}$ and then harvested for the apoptosis assay as described below.

Apoptosis assay. Cells $\left(1 \times 10^{5}\right)$ were suspended in $100 \mu 1$ binding buffer prior to addition of $5 \mu \mathrm{l}$ Annexin $\mathrm{V}$ and $5 \mu \mathrm{l}$ propidium iodide (PI) and incubation for $15 \mathrm{~min}$ at room temperature in the dark according to the manufacturer's instructions (BD Biosciences). Cells were then subjected to flow cytometric analysis to measure the rate of apoptosis (\%) with a Beckman Coulter EPICS Altra II cytometer (Beckman Coulter Inc., Brea, CA, USA).

Animal experiments. Adult female inbred Wistar rats (weight, $250 \pm 20 \mathrm{~g}$ ) were provided by the Experimental Animal Center of the Jilin University. The rats were anesthetized by intraperitoneal injection of pentobarbital $(40 \mathrm{mg} / \mathrm{kg})$, a midline incision was made on the back region and a laminectomy was performed aseptically at the T8-T12 level. The spinal cord was subjected to a $10 \mathrm{~g}$ weight-drop impact from a height of $5 \mathrm{~cm}$, which was repeated six times. The rats demonstrating severe loss of locomotor movement in the hind legs were randomly assigned to three treatment groups ( $n=20$ per group). Saline $(5 \mu \mathrm{l})$ or an identical volume of saline containing $1 \times 10^{5} \mathrm{SCs}$ or SCs-pEGFP-N1/GDNF was slowly injected into the site of the SCI, respectively. At each of the indicated time-points (seven and 15 days following cell injection), 10 rats (per group) were randomly sacrificed, and a $1-\mathrm{cm}$ long segment of the spinal cord was dissected between the cranial $(1 \mathrm{~cm})$ and caudal $(1 \mathrm{~cm})$ area of the injury epicenter. Each sample was split into two sections: One section was fixed with $10 \%$-buffered formalin and the other one was stored at $-80^{\circ} \mathrm{C}$ for further analysis.

Histological, immunocytochemical and immunohistochemical analyzes. Cells were fixed with $4 \%$ paraformaldehyde. Formalin-fixed spinal cord specimens were transferred to $70 \%$ ethanol and subsequently paraffin-embedded and sectioned. The sections $(5 \mu \mathrm{m})$ were stained with hematoxylin and eosin (H\&E) and examined under light microscopy (Eclipse TE2000-U equipped with an attached SXM1200F digital camera; Nikon Corporation, Tokyo, Japan). Cells or tissue sections were blocked with $3 \%$ biotinylated bovine serum albumin (BSA) and incubated with anti-rat Bcl-2 and Bax antibodies (Abs; Santa Cruz Biotechnology, Inc., Dallas, TX, USA), and then subsequently incubated for $30 \mathrm{~min}$ with appropriate secondary Abs (SP kit; Zhongshan Biotechnology Co., Beijing, China), and developed with Sigma FAST 3,3'-diaminobenzidine and $\mathrm{CoCl}_{2}$ enhancer tablets (Sigma-Aldrich). The slides were mounted and examined under a microscope.

Western blot analysis. Western blot analysis was performed as previously described (26). Briefly, cells or tissues were homogenized in protein lysate buffer and debris was removed by centrifugation. Protein samples $(20 \mu \mathrm{g})$ were resolved on polyacrylamide SDS gels and electrophoretically transferred to polyvinylidene difluoride membranes (Pierce PVDF 



\section{G}

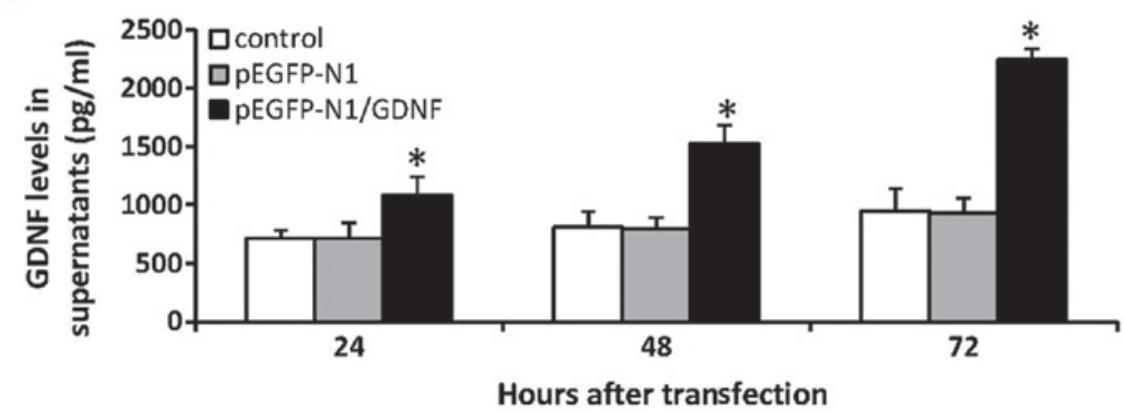

Figure 1. Genetically modified SCs express GDNF. (B and E) Rat RSC96 SCs were transfected with pEGFP-N1 or (C and F) pEGFP-N1/GDNF and transfectants were selected by co-culturing the cells with G418. (A and D) Untransfected cells served as controls. (A-C) Expression of EGFP was detected under a fluorescence microcope and expression of GDNF was detected by immunostaining the cells with an anti-GDNF antibody. (G) The cells from the abovementioned experiments were further cultured for 24, 48 and $72 \mathrm{~h}$, and the levels of GDNF in the supernatants were assessed using the ELISA. "Indicates a significant increase compared with the control or pEGFP-N1-transfected cells at the respective time-points. SC, Schwann cells; GDNF, glial cell line-derived neurotrophic factor; EGFP, enhanced green fluorescent protein; pEGFP-N1/GDNF, GDNF-expressing plasmid; pEGFP-N1, empty vector.

membranes, Thermo Fisher Scientific Inc., Rockford, IL USA). The membranes were blocked with 3\% BSA overnight, incubated with primary Abs (anti-rat Bax, Bcl-2 and $\beta$-actin; Santa Cruz Biotechnology, Inc., Dallas, TX, USA), then with an alkaline phosphatase-conjugated secondary $\mathrm{Ab}$ (AP kit; Zhongshan Golden Bridge Biotechnology Co., Ltd., Beijing, China), and immunoreactivity was visualized with 5-bromo-4-chloro-3-indolyl phosphate/nitro-blue tetrazolium chloride (BCIP/NBT Substrate Kit; Tiangen Biotech Co., Ltd., Beijing, China) according to the manufacturer's instructions.

Reverse transcription-polymerase chain reaction (RT-PCR) analysis. Total RNA was extracted from spinal cord homogenates using TRIzol ${ }^{\circledR}$ (Life Technologies) according to the manufacturer's instructions. The quality and amount of RNA were determined. RNA $(1 \mu \mathrm{g})$ was reverse-transcribed using a cDNA Synthesis kit (Takara Bio, Inc., Shiga, Japan) at $30^{\circ} \mathrm{C}$ for $5 \mathrm{~min}, 50^{\circ} \mathrm{C}$ for $30 \mathrm{~min}$, followed by $95^{\circ} \mathrm{C}$ for $5 \mathrm{~min}$. The cDNA was used as a template for PCR amplification under the following conditions: 30 cycles at $94^{\circ} \mathrm{C}$ for $30 \mathrm{sec}, 58^{\circ} \mathrm{C}$ for 30 sec and $72^{\circ} \mathrm{C}$ for $30 \mathrm{sec}$, with the following pairs of primers: 5'-CCCTGGCATCT TCTCCTTCC-3' and 5'-CAT CCCAGCCTCCGTTATCC-3' to generate a $446 \mathrm{bp}$ product of Bcl-2 mRNA; 5'-TTGTTACAGGGTTTCATCCAGG-3' and
5'-GAGTCCGTGTCCACGTCAG-3' to generate a $185 \mathrm{bp}$ product of Bax mRNA; and 5'-GTCAGGTCATCACTATCGG CAAT-3' and 5'-AGAGGTCTTTACGGATGTCAACGT-3' to generate a $147 \mathrm{bp}$ product of $\beta$-actin (served as an internal control). The products of the PCR were subjected to $2 \%$ agarose gel electrophoresis, and the density of each band was evaluated using the gel image analyzer (ultraviolet ChemiDOC; Bio-Rad, Hercules, CA, USA). The relative density of mRNA was calculated using the following formula: Band density/ $\beta$-actin band density.

Statistical analysis. All values are expressed as the mean \pm standard deviation. One-way analysis of variance was used to evaluate statistical significance. Data were analyzed using SPSS 15.0 (SPSS, Inc., Chicago, IL, USA). P<0.05 was considered to indicate a statistically significant difference.

\section{Results}

Expression of GDNF by genetically modified SCs. The RS96 SCs were transfected with pEGFP-N1 or pEGFP-N1/GDNF expression vectors and selected by co-culturing with G418. The untreated SCs exhibited no green fluorescence (Fig. 1A), whereas SCs transfected with pEGFP-N1 (Fig. 1B) or 


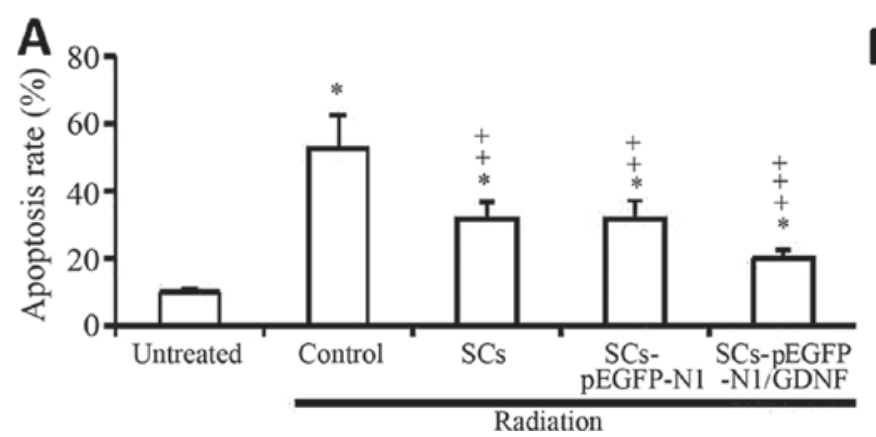

B

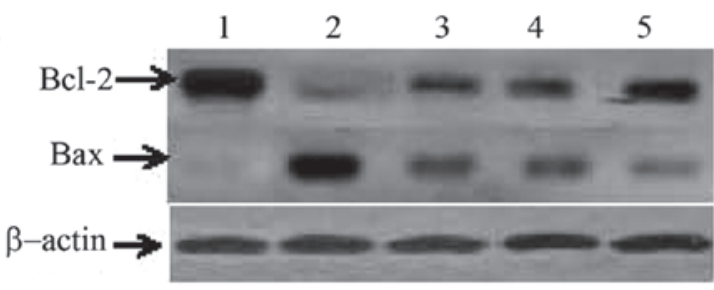

Figure 2. Co-culture with genetically modified SCs attenuates apoptosis of rat primary spinal neurons induced by radiation. Neuronal cells were exposed to radiation at $100 \mathrm{cGy} / \mathrm{min}$ for $24 \mathrm{~h}$, then co-cultured without SCs (control) or with SCs, SCs-pEGFP-N1 or SCs-pEGFP-N1-GDNF for $24 \mathrm{~h}$. (A) Untreated cells served as the negative control. The apoptosis of neurons was detected by flow cytometry. "Indicates a significant increase compared with untreated cells; ${ }^{\star} \operatorname{sig}$ nificant reduction compared with the control cells; or "significant reduction compared with the co-cultured SCs with SCs-pEGFP-N1. (B) Expression of Bcl-2 and Bax in untreated cells (lane 1), control cells exposed to radiation (lane 2), cells co-cultured with SCs (lane 3), SCs-pEGFP-N1 (lane 4) and SCs-pEGFP-N1GDNF (lane 5). SCs, Schwann cells; GDNF, glial cell line-derived neurotrophic factor; EGFP, enhanced green fluorescent protein; pEGFP-N1/GDNF, GDNF-expressing plasmid; pEGFP-N1, empty vector; Bcl-2, B-cell lymphoma 2; Bax, Bcl-2-associated x.
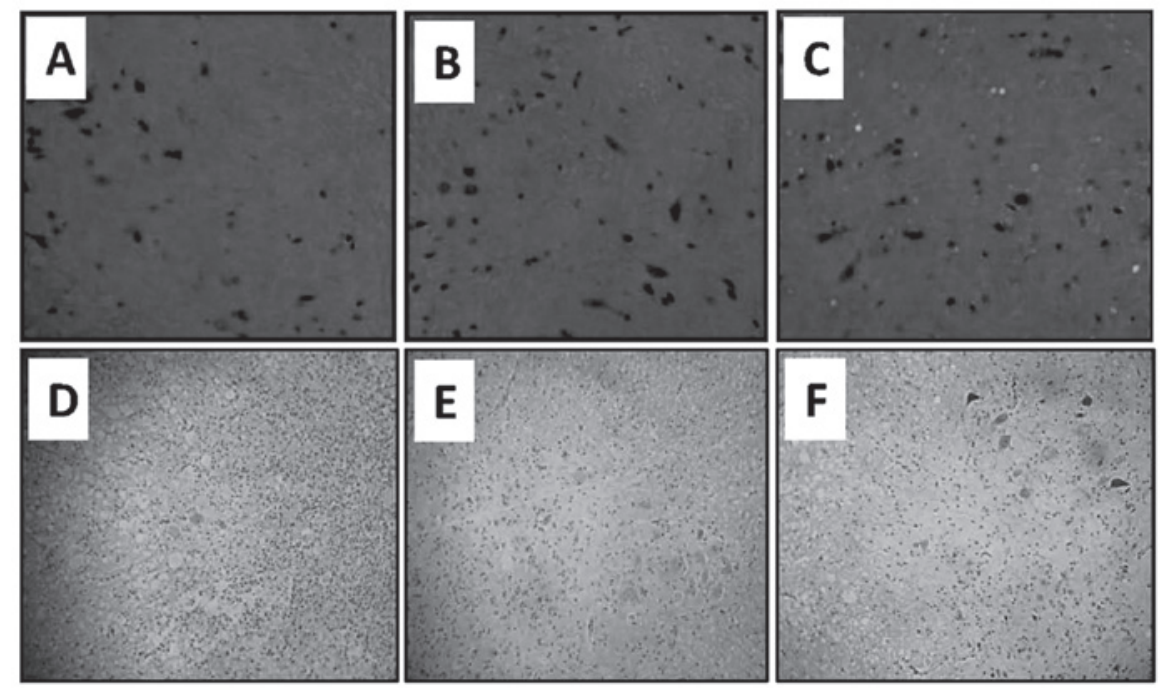

Figure 3. Injection of genetically modified SCs attenuates SCI. The spinal cords were obtained from (A) untreated rats, (B) rats with SCI injected with SCs or (C) SCs-pEGFP-N1-GDNF after 15 days and examined under a fluorescence microscope. The adjacent sections were stained with hematoxylin and eosin under light microscopy. The photographs obtained from fluorescence and light microscopy were merged using Photoshop software. Photographs display spinal cord sections obtained from rats with SCI (D) without treatment, (E) injected with SCs or (F) injected with SCs-pEGFP-N1-GDNF. SCs, Schwann cells; SCI, spinal cord injury; GDNF, glial cell line-derived neurotrophic factor; EGFP, enhanced green fluorescent protein; pEGFP-N1/GDNF, GDNF-expressing plasmid; pEGFP-N1, empty vector.

pEGFP-N1/GDNF (Fig. 1C) exhibited positive fluorescence under a fluorescence microscope. Immunocytochemical analysis performed with an anti-GDNF Ab revealed that untreated cells (Fig. 1D) and cells transfected with pEGFP-N1 (Fig. 1E) showed weak expression levels of GDNF, while cells transfected with pEGFP-N1/GDNF (Fig. 1F) showed a higher expression of GDNF. The above 3 types of cells were further cultured and the supernatants were collected and harvested to measure the concentrations of GDNF using ELISA. As shown in Fig. 1G, following culture for 24,48 and $72 \mathrm{~h}$ the levels of GDNF in the supernatant of SCs-pEGFP-N1/GDNF were significantly higher than those of unmodified SCs or SCs-pEGFP-N1. In addition, the levels of GDNF in the supernatant of SCs-pEGFP-N1/GDNF increased in a time-dependent manner, while GDNF levels remained similar in the supernatants of unmodified SCs or SCs-pEGFP-N1.
Genetically modified SCs inhibit apoptosis of rat primary spinal neurons induced by radiation. Primary spinal neuronal cells were collected from Wistar rats and exposed to radiation followed by co-culturing with unmodified SCs, SCs-pEGFP-N1 or SCs-pEGFP-N1/GDNF for $24 \mathrm{~h}$. Flow cytometric analysis was used to measure the rate of apoptosis. It was found that exposure to radiation significantly increased the rate of apoptosis in neurons $(53.2 \pm 9.5 \%)$ compared with that of the untreated neurons $(9.8 \pm 1.1 \%)(\mathrm{P}<0.001)$ (Fig. 2A). In addition, exposure to radiation followed by co-culture with unmodified SCs or SCs-pEGFP-N1 significantly attenuated apoptosis $(31.9 \pm 5.0$ or $32.3 \pm 5.1 \%$, respectively; $\mathrm{P}<0.05)$. However, exposure to radiation followed by co-culture with SCs-EGFP-N1/GDNF significantly reduced the rate of apoptosis $(20.1 \pm 2.9 \%)$, which was lower than that of the controls $(\mathrm{P}<0.001)$ and significantly lower than that of cells co-cultured with unmodified SCs or SCs-pGEFP-N1 (P<0.05) (Fig. 2A). 



Figure 4. Injection of genetically modified SCs regulated Bcl-2 and Bax expression in the spinal cords. (A-H) Spinal cords were obtained from (A and E) normal rats, (B and F) untreated rats with SCI, (C and G) rats with SCI injected with SCs and (D and H) rats with SCI injected with SCs-pEGFP-N1-GDNF 7 days following SCI. Spinal cords were sectioned, immunostained with (A-D) anti-Bcl-2 and (E-H) anti-Bax antibodies and examined under a microscope. (I and J) Expression of Bcl-2 and Bax protein were detected using western blot analysis in the spinal cords of (lane 1) normal rats, (lane 2) untreated rats with SCI, (lane 3) rats with SCI injected with SCs and (lane 4) rats with SCI injected with SCs-pEGFP-N1-GDNF. (J) The density of each band of protein was measured and compared with that of the internal control, $\beta$-actin. (K and I) Expression of Bcl-2 and Bax mRNA was detected with reverse transcriptionpolymerase chain reaction in spinal cords of (lane 1) normal rats, (lane 2) untreated rats with SCI, (lane 3) rats with SIC injected with SCs and (lane 4) rats with SIC injected with SCs-pEGFP-N1-GDNF. (L) The density of each band of mRNA was measured and compared with that of the internal control, $\beta$-actin. "Indicates significant difference from the normal rats; "significant difference from the untreated rats with SCI, and "significant difference from the rats with SCI treated with SCs. SCs, Schwann cells; GDNF, glial cell line-derived neurotrophic factor; EGFP, enhanced green fluorescent protein; pEGFP-N1/GDNF, GDNF-expressing plasmid; pEGFP-N1, empty vector; Bcl-2, B-cell lymphoma 2; Bax, Bcl-2-associated x.

Western blot analysis was used to examine the changes of Bcl-2 and Bax expression in the abovementioned cells. As shown in Fig. 2B, exposure to radiation downregulated the expression of $\mathrm{Bcl}-2$ and upregulated the expression of $\mathrm{Bax}$, and thus reduced the ratio of Bcl-2 to Bax. However, co-culture with unmodified SCs, SCs-pEGFP-N1 or SCs-pEGFP-N1/GDNF inhibited the changes in expression levels of Bcl-2 and Bax induced by radiation. Furthermore, co-culture with SCs-pEGFP-N1/GDNF showed the strongest effects in attenuating the alteration of Bcl-2 and Bax expression induced by radiation.
Transplantation of SCs attenuates spinal cord injury. The survival rates of SCs that were injected into the spinal cord of rats were examined. Normal Wistar rats were injected with unmodified SCs or SCs-pEGFP-N1/GDNF into the spinal cord. The unmodified SCs or SCs-pEGFP-N1/GDNF were harvested after 15 days, sectioned and examined by fluorescence microscopy; the adjacent sections were stained with H\&E and examined by light microscopy. The images captured through the fluorescence and light microscopes were merged using Photoshop software (Adobe Systems, San Jose, CA, USA). Representative images obtained from the spinal 
cord sections of normal control rats (Fig. 3A) and rats injected with unmodified SCs (Fig. 3B) demonstrated no fluorescence. However, the analysis of the spinal cord sections from rats injected with SCs-pEGFP-N1/GDNF revealed the presence of fluorescent cells (Fig. 3C), indicating that following transplantation, SCs survived in the spinal cords for $\sim 15$ days.

The effects of SC-transplantation on SCI were examined. The rats with SCI were randomly injected with saline (control) or $1 \times 10^{5}$ SCs or SCs-pEGFP-N1/GDNF into the spinal cords, and then randomly sacrificed after seven and 15 days. The spinal cords were harvested and subjected to histological analysis. Histological analysis of the central gray matter of SCI demonstrated hemorrhage, cell swelling, blurring Nissl bodies, pyknotic nuclei in several cells and infiltration of inflammatory cells. However, transplantation of SCs attenuated the histological changes, and SCs-pEGFP-N1/GDNF further inhibited the histological alterations. Representative images were captured from H\&E-stained spinal cord sections in untreated rats with SCI (Fig. 3D), rats with SCI treated with SCs (Fig. 3E) and rats with SCI treated with SCs-pEGFP-N1/GDNF (Fig. 3F) 15 days following cell transplantation.

Regulation of Bcl-2 and Bax expression in the spinal cords. The spinal cords of rats were subjected to immunohistochemical analysis with anti-Bcl-2 and anti-Bax Abs. As shown in Fig. 4A-H, SCI induced downregulation of Bcl-2 and upregulation of Bax, thus resulting in a reduced ratio of $\mathrm{Bcl}-2$ to $\mathrm{Bax}$. However, injection of SCs or SCs-pEGFP-N1/GDNF attenuated this change, resulting in increased expression of Bcl-2 and decreased expression of Bax, compared with that of untreated rats with SCI. This altered expression of Bcl-2 and Bax was further confirmed by western blot analysis detecting protein expression (Fig. 4I and J) and by RT-PCR detecting mRNA expression (Fig. 4K and L). Furthermore, quantification of the density of the bands in the western blots indicated that there was a significant difference in Bcl-2 and Bax at the protein and mRNA levels in SCs and SCs-pEGFP-N1/GDNF-treated SCI rats. This suggests that SCs-pEGFP-N1/GDNF may have marked anti-apoptotic activity against SCI-induced apoptosis compared with that of unmodified SCs due to its effects on the apoptosis-associated proteins Bcl-2 and Bax.

\section{Discussion}

The combination of SC transplantation and gene therapy is potentially one of the most powerful strategies to promote the recovery of the central nervous system (CNS), as SCs are involved in the repair of traumatic injury and demyelination in the spinal cord and other sections of the CNS. For example, SCs support axonal regeneration by secreting growth factors (27), providing a permissive extracellular matrix, secreting growth-promoting adhesion molecules and filling cystic cavities following SCI (28). Even when a biological conduit is used to bridge a new defect, the conduit is soon colonized by a number of SCs that form a pathway for regrowing axons. $\mathrm{SC}$ transplantation has been performed in various models of SCI, resulting in functional recovery $(29,30)$, and has more recently been applied in clinical trials of SCI (31). The present study demonstrated that injection of unmodified SCs inhibited SCI-induced apoptosis and attenuated SCI.
In addition, there is increasing interest in utilizing the regenerative properties of SCs, as SCs are a potential vector to introduce therapeutic genes for nerve repair. A promising option to foster nerve repair entails the genetic modification of autologous SCs either in vivo or in vitro, followed by reimplantation into the same individual for clinical application. Although viral expression vectors are the most efficient method to introduce transgenes into sites of SCI, their immunological response, safety issues and toxicity preclude their use in humans (32). Plasmid DNA has low immunogenicity, viral infection risks and is easy to propagate on a large scale at a high quality. The major limitation of the use of plasmid DNA as a vector is its low efficiency for gene delivery. The present study genetically modified rat RS96 SCs in vitro by transfection with the GDNF gene. The genetically modified SCs, which were selected with antibiotics, expressed high levels of GDNF in a time-dependent manner. Notably, following transplantation, SCs survived for $\sim 15$ days in the spinal cords of rats. Therefore, the SCs were able to support the recovery of SCI by guiding regenerating axons and providing trophic factors, in particular, high levels of GDNF.

GDNF was originally identified as a potent trophic factor for midbrain dopaminergic neurons, and was subsequently found to have the ability to support the survival of motor neurons both in vitro and in vivo. When applied to the spinal cord, GDNF exerts trophic effects on corticospinal neurons and promotes their long-term survival following axotomy (33). Administration of recombinant GDNF has been shown to improve behavioral outcome, neuronal survival and sprouting of fibers following SCI $(18,20)$. However, currently recombinant human GDNF protein is made from E. coli, insect cells or Pichia pastoris systems, which are unable to produce a mammalian glycosylation pattern (34). The clinical application of the GDNF protein has been hindered by high dose requirements, manufacturing constraints and its relative instability. However, transplantation of genetically modified cells producing GDNF represents an alternative method.

It has been demonstrated that GDNF inhibited retinoic acid-induced apoptosis in CHP134 neuroblastoma cells (35), attenuated ethanol-induced apoptosis and necrosis in SK-N-SH neuroblastoma cells (36), prevented ethanol-induced B92 glial cell death via the phosphoinositide-3-kinase/serine threonine protein kinase and mitogen-activated protein kinase/extracellular signal-regulated kinase signaling pathways (37), and protected against aluminum-induced apoptosis in rabbit brains by upregulating Bcl-2 and B-cell lymphoma extra large protein expression (38). The members of the Bcl-2 family are the most prominent regulators of apoptosis in various cell types, including cancer cells (39). Bcl-2, located on the mitochondrial membrane, is a proapoptotic protein, while Bax directly binds to Bcl-2 and inhibits its function (40). The present study demonstrated that genetically modified SCs producing GDNF inhibited the downregulation of Bcl-2 and the upregulation of Bax, which had been induced by radiation in vitro and by SCI in vivo. In the in vitro assays, a transwell membrane was used to prevent direct contact of SCs and neurons, which further confirms the protective effects of GDNF.

In conclusion, the present study demonstrated that genetically modified SCs producing high levels of GDNF attenuated SCI through anti-apoptotic activity. The engineered SCs were 
characterized by their ability to express and secrete biologically active GDNF, which inhibited radiation-induced apoptosis of primary spinal neurons by upregulating the expression of Bcl-2 and downregulating the expression of Bax in vitro. Following SC implantation into the spinal cord of adult rats with SCI induced by weight-drop impact, the modified SCs survived in the spinal cords and expressed high protein and mRNA levels of GDNF for $\sim 15$ days. Transplantation of modified SCs attenuated SCI and altered the expression of the apoptosis-associated proteins Bcl-2 and Bax, which was in accordance with the in vitro observations. The present study suggested that the growth-promoting properties of SCs were able to be significantly improved when these cells were genetically modified to secrete high levels of GDNF. The genetic engineering of SCs may provide novel possibilities for future clinical applications in SCI.

\section{References}

1. Qiao F, Atkinson C, Song H, Pannu R, Singh I and Tomlinson S: Complement plays an important role in spinal cord injury and represents a therapeutic target for improving recovery following trauma. Am J Pathol 169: 1039-1047, 2006.

2. Karimi-Abdolrezaee S, Eftekharpour E, Wang J, Morshead CM and Fehlings MG: Delayed transplantation of adult neural precursor cells promotes remyelination and functional neurological recovery after spinal cord injury. J Neurosci 26: 3377-3389, 2006

3. Fawcett J: Repair of spinal cord injuries: where are we, where are we going? Spinal Cord 40: 615-623, 2002.

4. Nandoe Tewarie RS, Hurtado A, Bartels RH, Grotenhuis A and Oudega M: Stem cell-based therapies for spinal cord injury. J Spinal Cord Med 32: 105-114, 2009.

5. Lankford KL, Imaizumi T, Honmou O and Kocsis JD: A quantitative morphometric analysis of rat spinal cord remyelination following transplantation of allogenic Schwann cells. J Comp Neurol 443: 259-274, 2002.

6. Schaal SM, Kitay BM, Cho KS, Lo TP Jr, Barakat DJ, Marcillo AE, Sanchez AR, Andrade CM and Pearse DD: Schwann cell transplantation improves reticulospinal axon growth and forelimb strength after severe cervical spinal cord contusion. Cell Transplant 16: 207-208, 2007.

7. Golden KL, Pearse DD, Blits B, Garg MS, Oudega M, Wood PM and Bunge MB: Transduced Schwann cells promote axon growth and myelination after spinal cord injury. Exp Neurol 207: 203-217, 2007

8. Lavdas AA, Franceschini I, Dubois-Dalcq M and Matsas R: Schwann cells genetically engineered to express PSA show enhanced migratory potential without impairment of their myelinating ability in vitro. Glia 53: 868-878, 2006.

9. Bunge MB: Bridging the transected or contused adult rat spinal cord with Schwann cell and olfactory ensheathing glia transplants. Prog Brain Res 137: 275-282, 2002.

10. Lavdas AA, Papastefanaki F, Thomaidou D and Matsas R: Schwann cell transplantation for CNS repair. Curr Med Chem 15 151-160, 2008.

11. Levi AD, Bunge RP, Lofgren JA, Meima L, Hefti F, Nikolics K and Sliwkowski MX: The influence of heregulins on human Schwann cell proliferation. J Neurosci 15: 1329-1340, 1995.

12. Lin LF, Doherty DH, Lile JD, Bektesh S and Collins F: GDNF: a glial cell line-derived neurotrophic factor for midbrain dopaminergic neurons. Science 260: 1130-1132, 1993.

13. Nandoe Tewarie RS, Hurtado A, Bartels RH, Grotenhuis A and Oudega M: Stem cell-based therapies for spinal cord injury. J Spinal Cord Med 32: 105-114, 2009.

14. Henderson CE, Phillips HS, Pollock RA, Davies AM, Lemeulle C, Armanini M, Simpson LC, Moffet B, Vandlen RA, Koliatsos VE and Rosenthal A: GDNF: a potent survival factor for motoneurons present in peripheral nerve and muscle. Science 266: 1062-1064, 1994.

15. Tomac A, Lindqvist E, Lin LF, Ögren SO, Young D, Hoffer BJ and Olson L: Protection and repair of the nigrostriatal dopaminergic system by GDNF in vivo. Nature 373: 335-339, 1995 .

16. Yan Q, Matheson C and Lopez OT: In vivo neurotrophic effects of GDNF on neonatal and adult facial motor neurons. Nature 373 341-344, 1995
17. Beck KD, Valverde J, Alexi T, Poulsen K, Moffat B, Vandlen RA, Rosenthal A and Hefti F: Mesencephalic dopaminergic neurons protected by GDNF from axotomy-induced degeneration in the adult brain. Nature 373: 339-341, 1995.

18. Ansorena E, Garbayo E, Lanciego JL, Aymerich MS and Blanco-Prieto MJ: Production of highly pure human glycosylated GDNF in a mammalian cell line. Int J Pharm 385: 6-11, 2010.

19. Cheng H, Wu JP and Tzeng SF: Neuroprotection of glial cell line-derived neurotrophic factor in damaged spinal cords following contusive injury. J Neurosci Res 69: 397-405, 2002.

20. Sharma HS: Post-traumatic application of brain-derived neurotrophic factor and glia-derived neurotrophic factor on the rat spinal cord enhances neuroprotection and improves motor function. Acta Neurochir Suppl 96: 329-334, 2006.

21. Guzen FP, De Almeida Leme RJ, de Andrade MS, de Luca BA and Chadi G: Glial cell line-derived neurotrophic factor added to a sciatic nerve fragment grafted in a spinal cord gap ameliorates motor impairments in rats and increases local axonal growth. Restor Neurol Neurosci 27: 1-16, 2009.

22. Zhang L, Ma Z, Smith GM, Wen X, Pressman Y, Wood PM and Xu XM: GDNF-enhanced axonal regeneration and myelination following spinal cord injury is mediated by primary effects on neurons. Glia 57: 1178-1191, 2009.

23. Piquilloud G, Christen T,Pfister LA, Gander B and Papaloizos MY: Variations in glial cell line-derived neurotrophic factor release from biodegradable nerve conduits modify the rate of functional motor recovery after rat primary nerve repairs. Eur J Neurosci 26: 1109-1117, 2007.

24. Lang AE, Gill S, Patel NK, Lozano A, Nutt JG, Penn R, Brooks DJ, Hotton G, Moro E, Heywood P, Brodsky MA, Burchiel K, Kelly P, Dalvi A, Scott B, Stacy M, Turner D, Wooten VG, Elias WJ, Laws ER, Dhawan V, Stoessl AJ, Matcham J, Coffey RJ and Traub M: Randomized controlled trial of intraputamenal glial cell line-derived neurotrophic factor infusion in Parkinson disease. Ann Neuro 59: 459-466, 2006.

25. Carnicella S and Ron D: GDNF, a potential target to treat addiction. Pharmacol Ther 122: 9-18, 2009.

26. Moreels M, Vandenabeele F, Deryck L and Lambrichts I: Radial glial cells derived from the neonatal rat spinal cord: morphological and immunocytochemical characterization. Arch Hictol Cytol 68: 361-369, 2005.

27. Sun X, Liu M, Wei Y, Liu F, Zhi X, Xu R and Krissansen GW: Overexpression of von Hippel-Lindau tumor suppressor protein and antisense HIF-1alpha eradicates gliomas. Cancer Gene Ther 13: 428-435, 2006.

28. Bampton ET and Taylor JS: Effects of Schwann cell secreted factors on PC12 cell neuritogenesis and survival. J Neurobiol 63: 29-48, 2005.

29. Afshari FT, Kwok JC, White L and Fawcett JW: Schwann cell migration is integrin-dependent and inhibited by astrocyte-produced aggrecan. Glia 58: 857-869, 2010.

30. Pearse DD, Sanchez AR, Pereira FC, Andrade CM, Puzis R, Pressman Y, Golden K, Kitay BM, Blits B, Wood PM and Bunge MB: Transplantation of Schwann cells and/or olfactory ensheathing glia into the contused spinal cord: Survival, migration, axon association, and functional recovery. Glia 55: 976-1000, 2007.

31. Takami T, Oudega M, Bates ML, Wood PM, Kleitman N and Bunge MB: Schwann cell but not olfactory ensheathing glia transplants improve hindlimb locomotor performance in the moderately contused adult rat thoracic spinal cord. J Neurosci 22: 6670-6681, 2002.

32. Saberi H, Moshayedi P, Aghayan HR, Arjmand B, Hosseini SK, Emami-Razavi SH, Rahimi-Movaghar V, Raza M and Firouzi M: Treatment of chronic thoracic spinal cord injury patients with autologous Schwann cell transplantation: an interim report on safety considerations and possible outcomes. Neurosci Lett 443: 46-50, 2008.

33. Zabner J, Ramsey BW, Meeker DP, et al: Repeat administration of an adenovirus vector encoding cystic fibrosis transmembrane conductance regulator to the nasal epithelium of patients with cystic fibrosis. J Clin Invest 97: 1504-1511, 1996.

34. Giehl KM, Schacht CM, Yan Q and Mestres P: GDNF is a trophic factor for adult rat corticospinal neurons and promotes their long-term survival after axotomy in vivo. Eur J Neurosci 9: 2479-2488, 1997

35. Cheng H, Wu JP and Tzeng SF: Neuroprotection of glial cell line-derived neurotrophic factor in damaged spinal cords following contusive injury. J Neurosci Res 69: 397-405, 2002. 
36. Guzen FP, de Almeida Leme RJ, de Andrade MS, de Luca BA and Chadi G: Glial cell line-derived neurotrophic factor added to a sciatic nerve fragment grafted in a spinal cord gap ameliorates motor impairments in rats and increases local axonal growth. Restor Neurol Neurosci 27: 1-16, 2009.

37. Gerngross TU: Advances in the production of human therapeutic proteins in yeasts and filamentous fungi. Nat Biotechnol 22: 1409-1414, 2004.

38. Takada N, Isogai E, Kawamoto T, Nakanishi H, Todo S and Nakagawara A: Retinoic acid-induced apoptosis of the CHP134 neuroblastoma cell line is associated with nuclear accumulation of p53 and is rescued by the GDNF/Ret signal. Med Pediatr Oncol 36: 122-126, 2001

39. McAlhany RE Jr, West JR and Miranda RC: Glial-derived neurotrophic factor (GDNF) prevents ethanol-induced apoptosis and JUN kinase phosphorylation. Brain Res Dev Brain Res 119: 209-216, 2000
40. Villegas SN, Njaine B, Linden R and Carri NG: Glial-derived neurotrophic factor $(\mathrm{GDNF})$ prevents ethanol $(\mathrm{EtOH})$ induced B92 glial cell death by both PI3K/AKT and MEK/ERK signaling pathways. Brain Res Bull 71: 116-126, 2006.

41. Ghribi O, Herman MM, Forbes MS, DeWitt DA and Savory J: GDNF protects against aluminum-induced apoptosis in rabbits by upregulating Bcl-2 and $\mathrm{Bcl}-\mathrm{XL}$ and inhibiting mitochondrial Bax translocation. Neurobiol Dis 8: 764-773, 2001.

42. Walensky LD: BCL-2 in the crosshairs: tipping the balance of life and death. Cell Death Differ 13: 1339-1350, 2006.

43. Yang J, Liu X, Bhalla K, Kim CN, Ibrado AM, Cai J, Peng TI, Jones DP and Wang X: Prevention of apoptosis by Bcl-2: release of cytochrome c from mitochondria blocked. Science 275: 1129-1132, 1997. 